\title{
SELECTIVE CATALYST SURFACE ACCESS THROUGH ATOMIC LAYER DEPOSITION
}

Samuel S. Hardisty, Shira Frank, Melina Zysler, Reut Yemini, Anya Muzikansky, Malachi Noked, David Zitoun*

Email:david.zitoun@,biu.ac.il

Table S1. V\%wt. of the samples after the ALD deposition process from ICP-AES

\begin{tabular}{cc} 
Sample & V\% wt. via ICP \\
\hline 36 ALD cycles (Pt/C) & 0.148 \\
72 ALD cycles (Pt/C) & 0.309 \\
144 ALD cycles (Pt/C) & 0.329 \\
720 ALD cycles $(\mathrm{Pt} / \mathrm{C})$ & 0.514 \\
72 ALD cycles $(\mathrm{C})$ & Not detected
\end{tabular}

Equation S1. Calculation of $\mathrm{V}_{2} \mathrm{O}_{5}$ coverage.

The density of $\mathrm{V}_{2} \mathrm{O}_{5}$ is $3.36 \mathrm{~g} \mathrm{~cm}^{-3}$.

As we don't know the direction of growth, we will use the largest lattice parameter of $\mathrm{V}$ which is $11.519 \AA .{ }^{1}$

This means that the surface area covered by one unit cell thickness of $\mathrm{V}_{2} \mathrm{O}_{5}$ is

$$
V_{\text {coverage }}=3.36 \times 1.1519 \times 10^{-7}=3.870 \times 10^{-7} \mathrm{~g} \mathrm{~cm}^{-2}
$$

The ECSA the Pt in the $50 \% \mathrm{Pt} / \mathrm{C}$ substrate is $24.8 \mathrm{~m}^{2} \mathrm{~g}^{-1}$, or $248000 \mathrm{~cm}^{2} \mathrm{~g}^{-1}$.

Therefore, the mass of $\mathrm{V}$ needed to cover the $\mathrm{Pt}$ of $1 \mathrm{~g}$ of the $50 \% \mathrm{Pt} / \mathrm{C}$ with 1 unit cell is:

$$
\frac{(50.942 \times 2) \times V_{\text {coverage }} \times E C S A \text { of Pt }}{(50.492 \times 2)+(15.999 \times 5)}=0.00096 \mathrm{~g}=0.096 \% \mathrm{wt} .
$$


Equation S2. Scherrer equation

$$
F W H M=\frac{K \lambda}{L \cos \theta}
$$

Where $K$ is the Scherrer constant, $\mathrm{L}$ is the crystal thickness, and $\theta$ is the peak position.

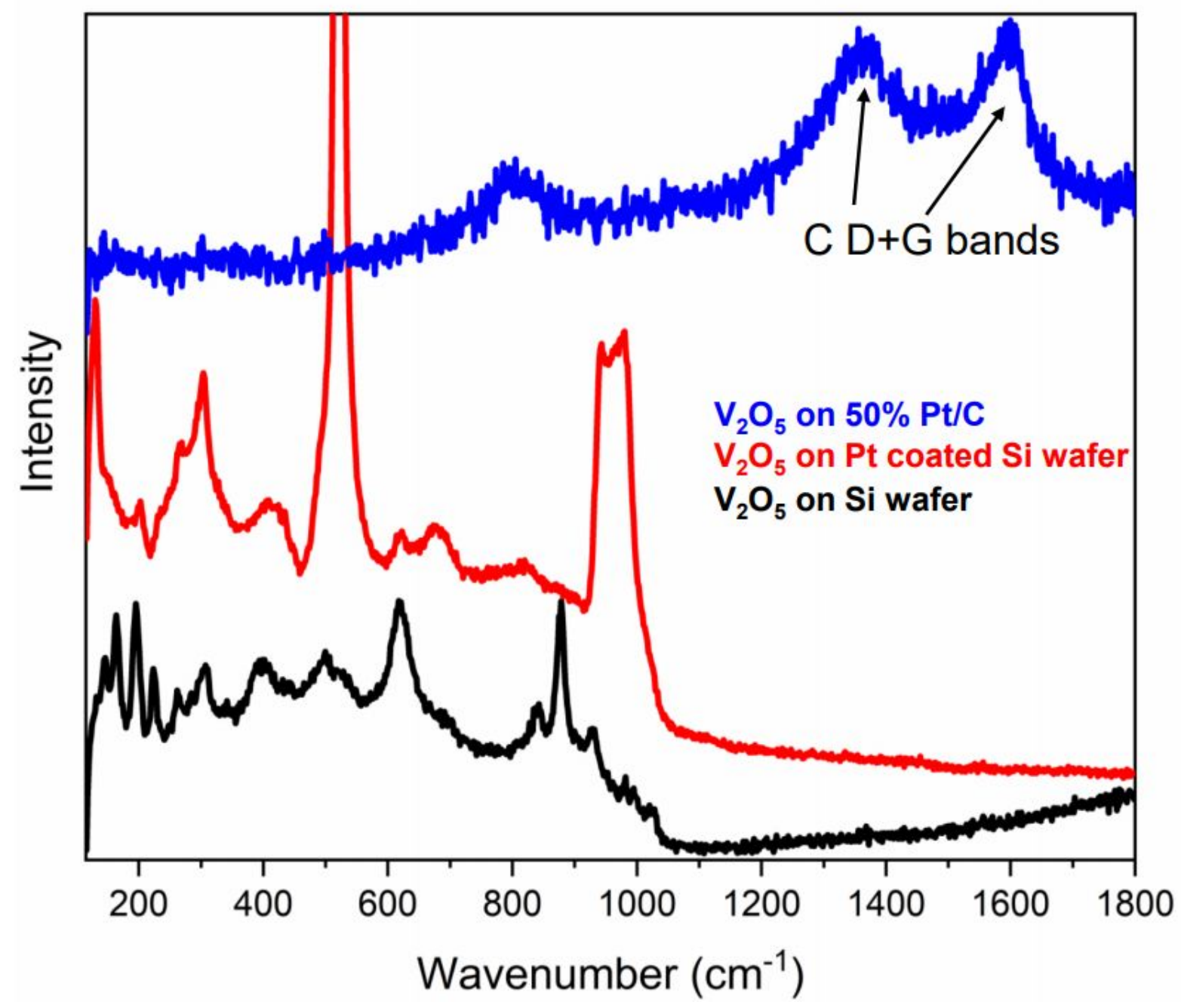

Figure S1. Raman spectra of $\mathrm{V}_{\mathrm{x}} \mathrm{O}_{\mathrm{y}}$ on $50 \% \mathrm{Pt} / \mathrm{C}$ (720 ALD cycles), Pt coated $\mathrm{Si}$ wafer and $\mathrm{Si}$ wafer. 
Table S2. Raman peaks and assignments for $\mathrm{V}_{2} \mathrm{O}_{5}$ deposition on Si wafer.

\begin{tabular}{cccc} 
Band & Assignment & Symmetry & Species \\
\hline 144 & $\mathrm{~T}_{\mathrm{y}}, \mathrm{R}_{\mathrm{z}}$ & $\mathrm{B}_{\mathrm{g}}$ & $\mathrm{V}_{2} \mathrm{O}_{5}$ \\
164 & $\mathrm{~T}, \mathrm{R}$ & $\mathrm{B}_{\mathrm{g}}, \mathrm{A}_{\mathrm{g}}$ & $\mathrm{V}_{2} \mathrm{O}_{5-\mathrm{x}}$ \\
195 & $\mathrm{~T}_{\mathrm{x}}, \mathrm{R}_{\mathrm{y}}, \delta\left(\mathrm{O}_{1} \mathrm{VO}_{3}\right)$ & $\mathrm{B}_{\mathrm{g}}, \mathrm{A}_{\mathrm{g}}$ & $\mathrm{V}_{2} \mathrm{O}_{5}$ \\
223 & & & $\mathrm{SiO}_{2}$ \\
262 & $\delta\left(\mathrm{O}_{1} \mathrm{VO}_{3}\right)$ & $\mathrm{B}_{\mathrm{g}}$ & $\mathrm{V}_{2} \mathrm{O}_{5}$ \\
301 & & & $\mathrm{Si}$ \\
399 & $\delta\left(\mathrm{VO}_{3} \mathrm{~V}\right)$ & $\mathrm{A}_{\mathrm{g}}$ & $\mathrm{V}_{2} \mathrm{O}_{5}$ \\
520 & & & $\mathrm{Si}$ \\
617 & & & $\mathrm{SiO}_{2}$ \\
670 & & & $\mathrm{SiO}_{2}$ \\
838 & & & $\mathrm{SiO}_{2}$ \\
880 & & & $\mathrm{SiO}_{2}$ \\
928 & & & $\mathrm{Si}$ \\
981 & & & $\mathrm{Si}$ \\
995 & $v\left(\mathrm{O}_{1}\right)$ & $\mathrm{A}_{\mathrm{g}}$ & $\mathrm{V}_{2} \mathrm{O}_{5}$ \\
1020 & $v\left(\mathrm{O}_{1}\right)$ & $\mathrm{A}_{\mathrm{g}}$ & $\mathrm{V}_{2} \mathrm{O}_{5-\mathrm{x}}$
\end{tabular}

Table S3. Raman peaks and assignments for $\mathrm{V}_{2} \mathrm{O}_{5}$ deposition on Pt coated Si wafer.

$\begin{array}{cccc}\text { Band } & \text { Assignment } & \text { Symmetry } & \text { Species } \\ 144 & \mathrm{~T}_{\mathrm{y}}, \mathrm{R}_{\mathrm{z}} & \mathrm{B}_{\mathrm{g}} & \mathrm{V}_{2} \mathrm{O}_{5} \\ 202 & \mathrm{~T}_{\mathrm{x}}, \mathrm{R}_{\mathrm{y}}, \delta\left(\mathrm{O}_{1} \mathrm{VO}_{3}\right) & \mathrm{B}_{\mathrm{g}}, \mathrm{A}_{\mathrm{g}} & \mathrm{V}_{2} \mathrm{O}_{5} \\ 235 & & & \mathrm{SiO}_{2} \\ 265 & \delta\left(\mathrm{O}_{1} \mathrm{VO}_{3}\right) & \mathrm{B}_{\mathrm{g}} & \mathrm{V}_{2} \mathrm{O}_{5} \\ 301 & & & \mathrm{Si} \\ 430 & \delta\left(\mathrm{VO}_{3} \mathrm{~V}\right) & \mathrm{A}_{\mathrm{g}} & \mathrm{V}_{2} \mathrm{O}_{5} \\ 520 & & & \mathrm{Si} \\ 622 & & & \mathrm{SiO}_{2} \\ 672 & & & \mathrm{SiO}_{2} \\ 819 & & & \mathrm{SiO}_{2} \\ 928 & & & \mathrm{Si} \\ 981 & & & \mathrm{Si}^{2}\end{array}$


Table S4. V 2p and Pt 4f XPS peak relative \%at. for the various samples.

\begin{tabular}{ccccc} 
Sample & $\mathbf{V}^{\mathbf{4}}$ & $\mathbf{V}^{\mathbf{5}^{+}}$ & $\mathbf{V}^{\mathbf{5}} / \mathbf{P t}$ & $\mathbf{P t}$ \\
\hline 0 ALD cycles & 0 & 0 & 0 & 100 \\
36 ALD cycles & 3.91 & 5.68 & 6.11 & 84.3 \\
72 ALD cycles & 5.55 & 6.28 & 6.27 & 81.9 \\
144 ALD cycles & 5.72 & 7.32 & 6.33 & 80.63 \\
720 ALD cycles & 6.01 & 8.56 & 5.91 & 79.52
\end{tabular}

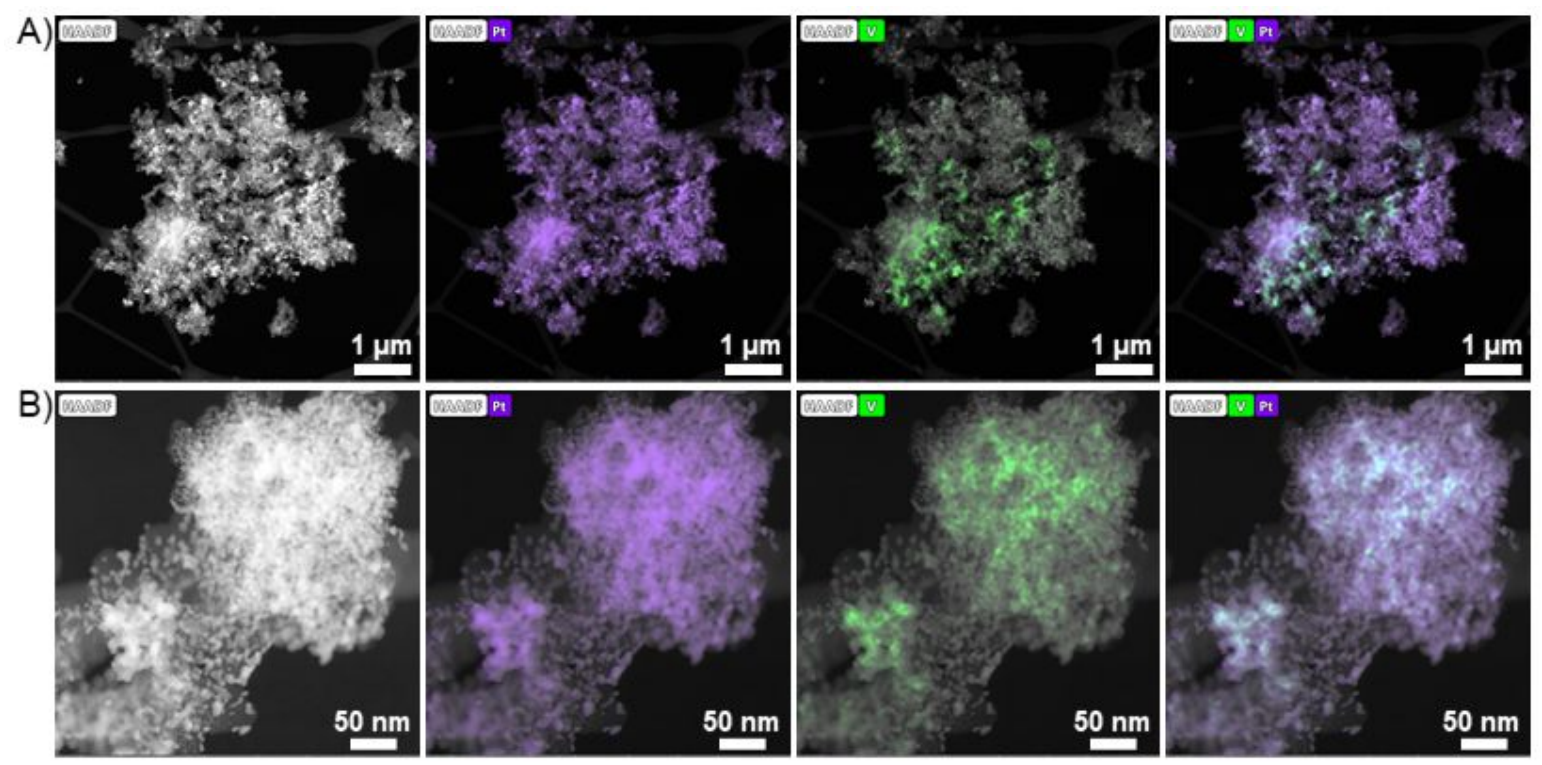

Figure S2. STEM micrographs with EDS mapping for the 36 ALD cycles (A) and 720 ALD cycles (B) samples. 


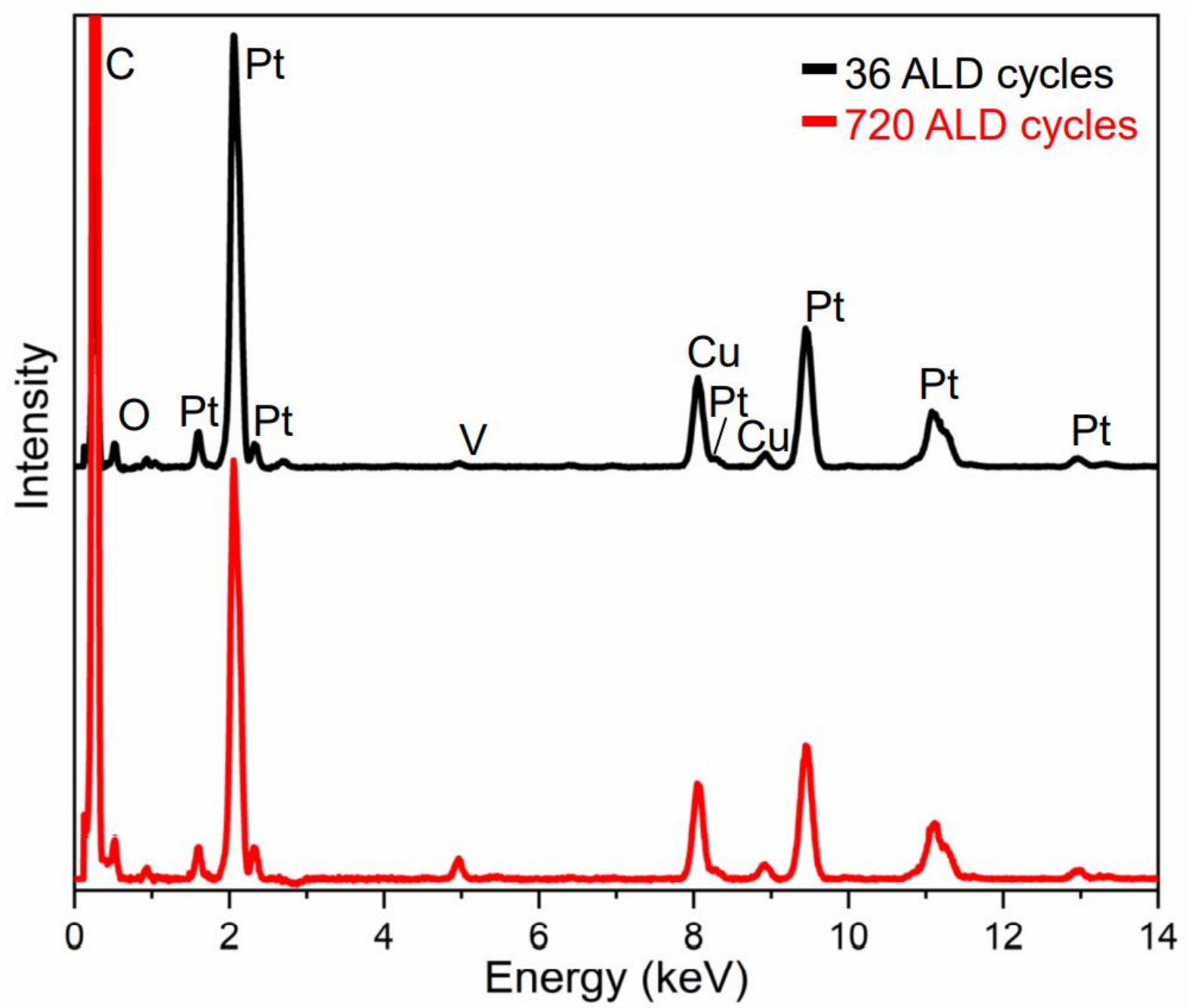

Figure S3. EDS spectra of $50 \% \mathrm{Pt} / \mathrm{C}$ after 36 and 720 ALD cycles. 


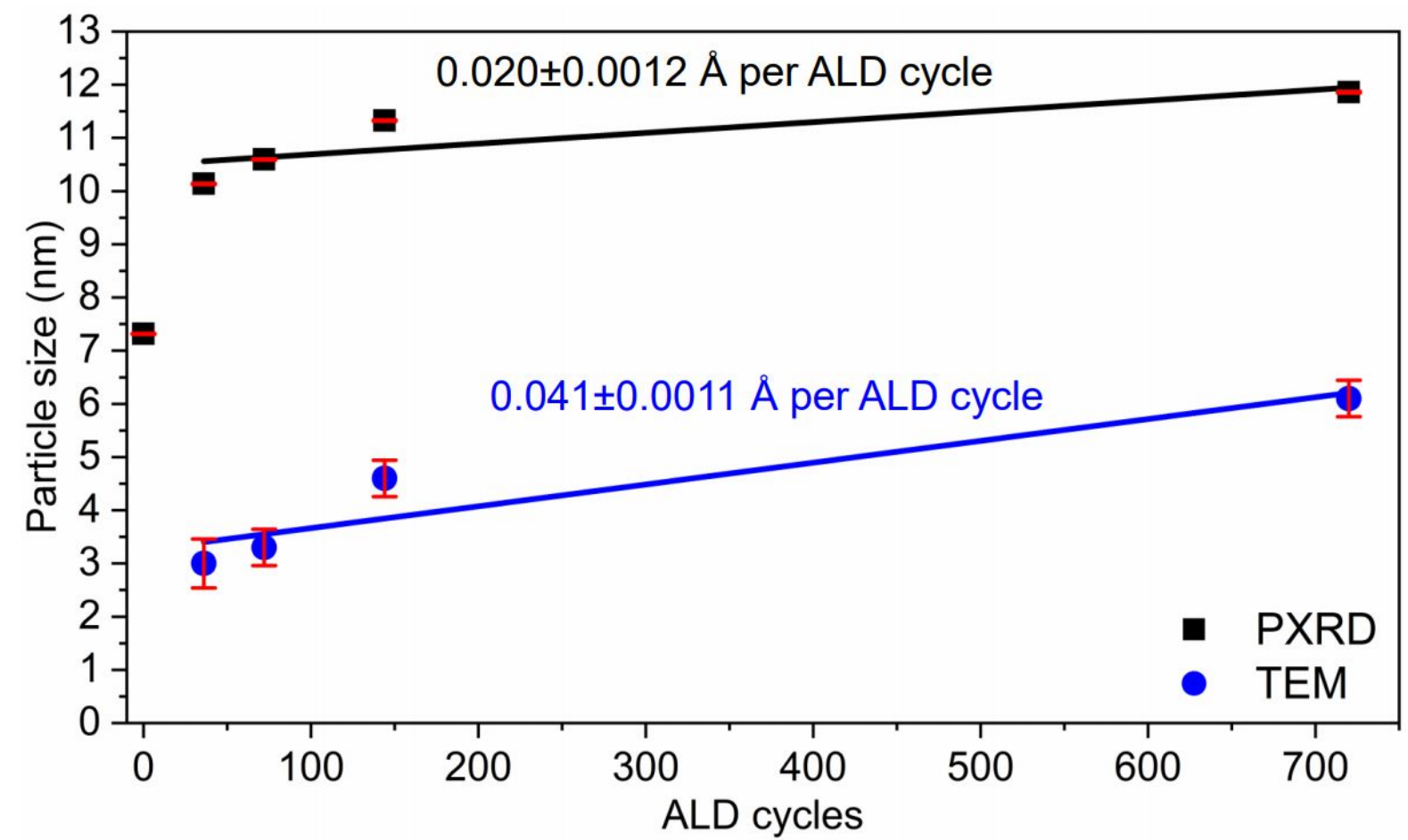

Figure S4. Comparison of particle sizes for the samples from PXRD $\left(\mathrm{R}^{2}=0.67\right)$ and TEM $\left(\mathrm{R}^{2}=0.87\right)$. Error bars show the standard deviation of the particle size for each sample.
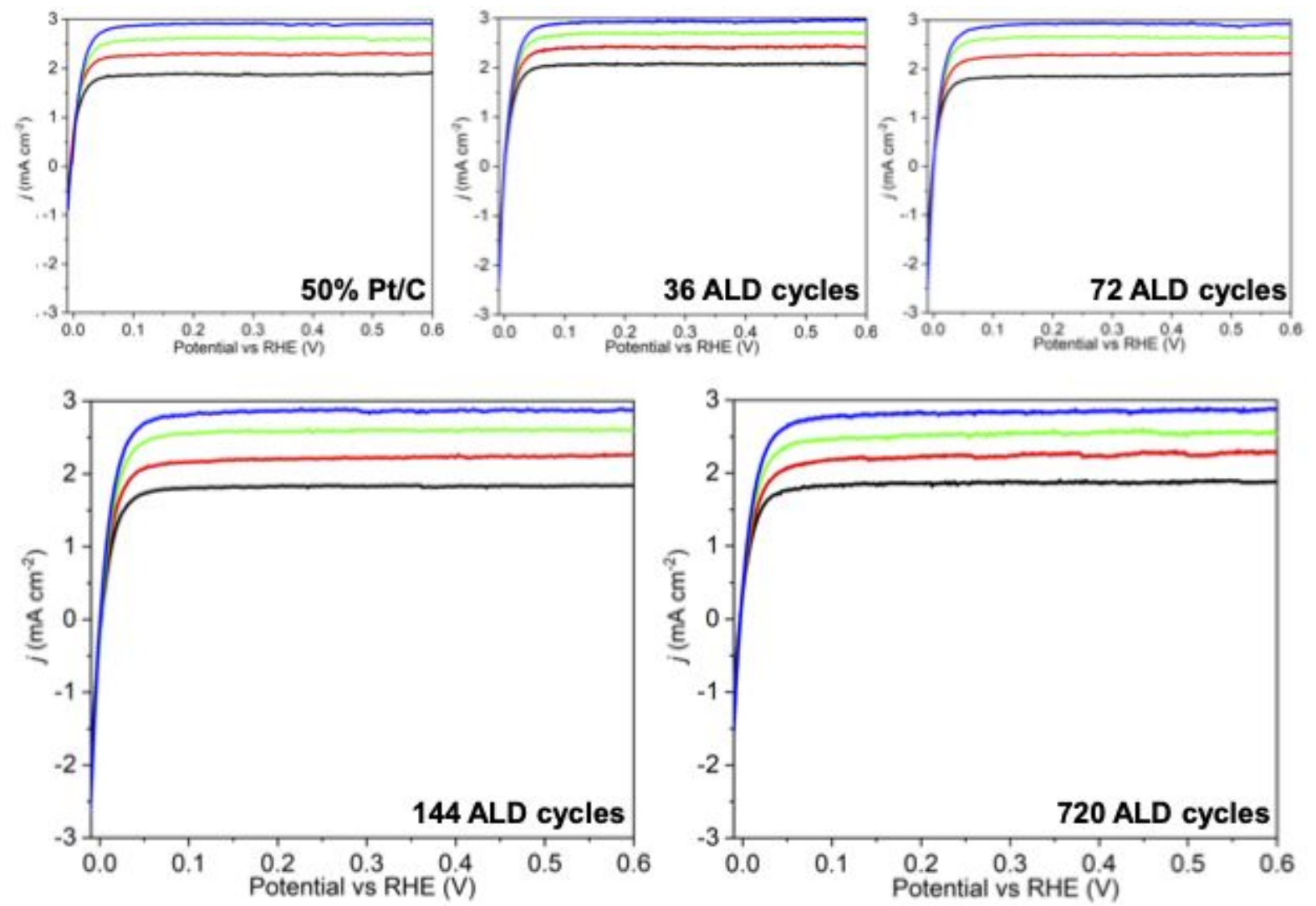

Figure S5. HOR polarization curves for the 5 samples at 625 (black), 900 (red), 1225 (green) and 1600 (blue) rpm in $0.1 \mathrm{M} \mathrm{HClO}_{4}$ at a scan rate of $5 \mathrm{mV} \mathrm{s}^{-1}$. 
Equation S3. Koutecky-Levich equation. ${ }^{2}$

$$
j_{\text {measured }}^{-1}=j_{k}^{-1}+j_{D}^{-1}
$$

Where: $j_{k}=$ kinetic current and $j_{D}=$ mass-transport limited current

Equation S4. Butler Volmer equation. ${ }^{2}$

$$
j_{k}=j_{0}\left[e^{(\alpha F / R T) \eta}-e^{(-(1-\alpha) F / R T) \eta}\right]
$$

Where: $j_{0}=$ exchange current density, $\alpha=$ transfer coefficient, $F=$ Faraday's constant, $R=$ universal gas constant, $T=$ temperature $(\mathrm{K})$ and $\eta=$ overpotential
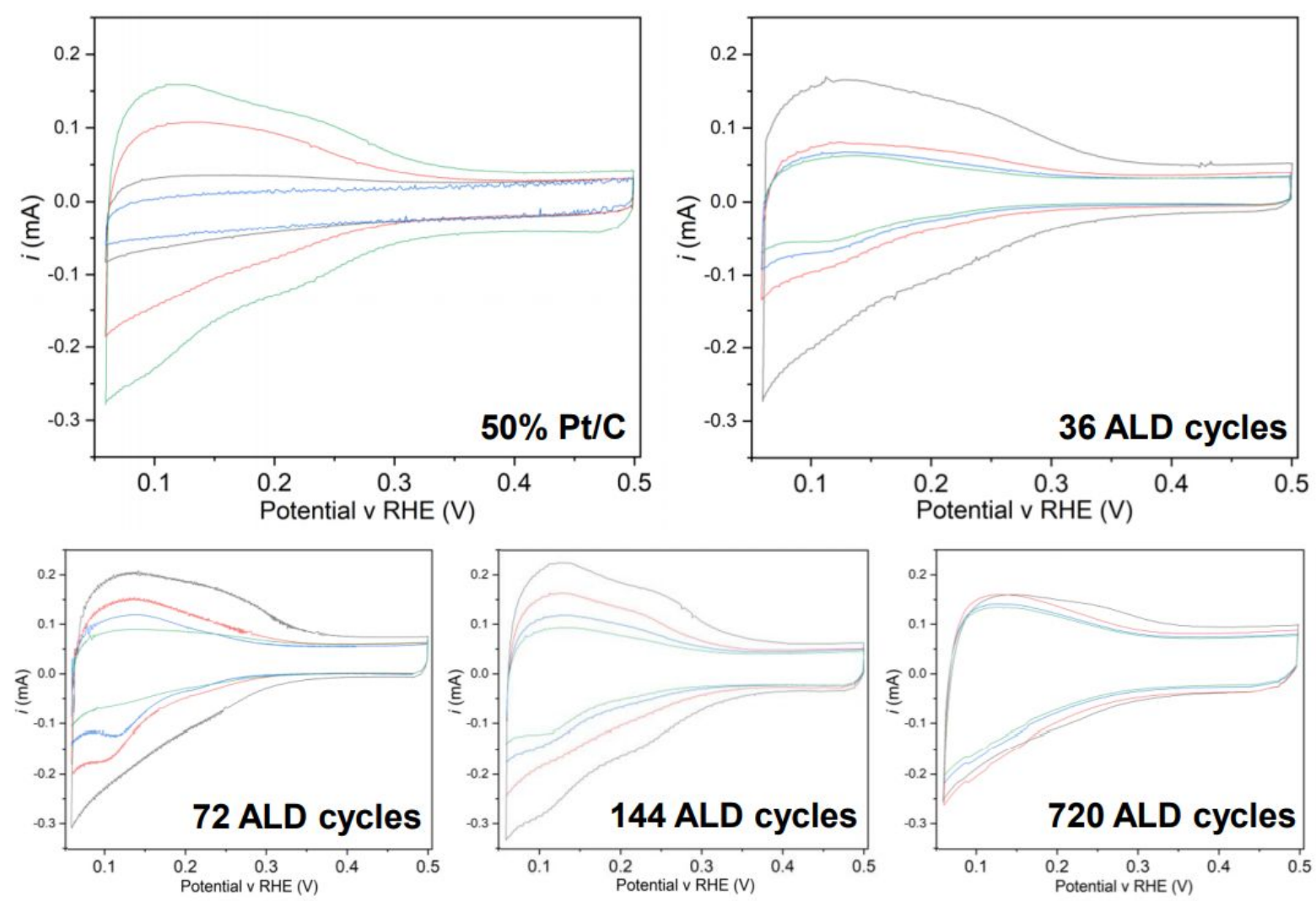

Figure S6. CVs of the different samples before and after soaking in $33 \% \mathrm{SOC}, 40{ }^{\circ} \mathrm{C} \mathrm{HBr}$ electrolyte (data from which figure 5A was calculated). 


\section{REFERENCES}

(1) Chakrabarti, A.; Hermann, K.; Druzinic, R.; Witko, M.; Wagner, F.; Petersen, M. Geometric and Electronic Structure of Vanadium Pentoxide: A Density Functional Bulk and Surface Study.

(2) Wei, C.; Rao, R. R.; Peng, J.; Huang, B.; Stephens, I. E. L.; Risch, M.; Xu, Z. J.; ShaoHorn, Y. Recommended Practices and Benchmark Activity for Hydrogen and Oxygen Electrocatalysis in Water Splitting and Fuel Cells. Adv. Mater. 2019, 31 (31), 1806296. https://doi.org/10.1002/ADMA.201806296. 